\title{
A narrative review of malnutrition in chronic liver disease
}

\author{
Soan Shin ${ }^{1}$, Dae Won Jun ${ }^{1,2}$, Waqar Khalid Saeed ${ }^{3}$, Dong Hee Koh ${ }^{4}$ \\ ${ }^{1}$ School of Medicine, Hanyang University, Seoul, South Korea; ${ }^{2}$ Department of Internal Medicine, School of Medicine, Hanyang University, Seoul, \\ South Korea; ${ }^{3}$ Department of Biomedical Sciences, Pak-Austria Fachhochschule: Institute of Applied Sciences and Technology, Mang, Haripur, \\ Pakistan; ${ }^{4}$ Department of Internal Medicine, Hallym University Dongtan Sacred Heart Hospital, Hwaseong-si, South Korea \\ Contributions: (I) Conception and design: DW Jun; (II) Administrative support: WK Saeed; (III) Provision of study materials or patients: S Shin; (IV) \\ Collection and assembly of data: S Shin; (V) Data analysis and interpretation: DH Koh; (VI) Manuscript writing: All authors; (VII) Final approval of \\ manuscript: All authors. \\ Correspondence to: Dae Won Jun, MD. Department of Internal Medicine, Hanyang University Hospital, Hanyang University College of Medicine, 222 - \\ 1, Wangsimni-ro, Seongdong-gu, Seoul 04763, South Korea. Email: noshin@hanyang.ac.kr; Dong Hee Koh. Department of Internal Medicine, Hallym \\ University Dongtan Sacred Heart Hospital, 7, Keunjaebong-gil, Hwaseong-si, Gyeonggi-do 445-907, South Korea. Email: dhkoh@hallym.or.kr.
}

\begin{abstract}
Interest in research on malnutrition is decreasing due to thoughts that the problem of malnutrition has been solved in an age of over-nourishment or obesity and defining malnutrition is not uniform. This study aimed to critically appraise the prevalence of malnutrition according to various diagnostic tools and proportion of severity used in previous studies. A literature review was performed using a total of 16 studies published between 1980 and 2020 regarding malnutrition in patients with chronic liver disease. Most of the analyzed studies were conducted before 2010, and only a few studies were conducted after 2010. Nutrition assessment tool (NAT) and nutrition screening tool (NST) to explain malnutrition were distinguished; however, there was no clear distinction between them. NST often used questionnaires while NST used various malnutrition measuring tools. Our results show that, in the age of over-nourishment, reduction in malnutrition in chronic liver disease still hasn't been significant. Malnutrition prevalence in studies published prior to 2,000 ranged between $13.3 \%$ and $85 \%$ (mean, 37.6\%), whereas that in studies published after 2,000 ranged between $13.3 \%$ and $78.5 \%$ (mean, 35.2\%). Malnutrition prevalence largely depends on the diagnostic tool and proportion of disease severity in the target population. The prevalence of malnutrition in patients with chronic liver diseases varies widely. This big difference is related to various diagnostic tools, mixed etiologies, and different disease severity in different studies. The prevalence of malnutrition was $36.4 \%(10-80.3 \%)$ in all patients with liver disease, 39.9\% (13.3-80.3\%) in compensated liver disease, and 44.1\% (26.7-93.6\%) in decompensated cirrhosis. Malnutrition prevalence was 38.2\% and $23.7 \%$ in alcoholism-related and hepatitis $\mathrm{C}$ virus (HCV)-related diseases, respectively. Malnutrition also largely depended on the judgement tool. Malnutrition prevalence according to the diagnostic tool was approximately 28-85\% for subjective global assessment (SGA), 30.8-78.5\% for anthropometric approach, and $21-80.3 \%$ for clinical judgment. It became similar over time.
\end{abstract}

Keywords: Malnutrition; liver; cirrhosis; hepatitis C virus (HCV); subjective global assessment (SGA); triceps skinfold thickness (TSF)

Submitted Jun 22, 2020. Accepted for publication Nov 27, 2020.

doi: 10.21037/atm-20-4868

View this article at: http://dx.doi.org/10.21037/atm-20-4868 


\section{Introduction}

Prevalence of malnutrition is common in patients with chronic liver diseases. Malnutrition increases mortality and aggravated disease course of liver diseases (1). Therefore, an accurate evaluation of a patient's nutritional status is important for the treatment and management of chronic liver disease. Various factors such as reduced intake, malabsorption, hypermetabolic status, bacterial overgrowth, and altered nutrient metabolism increases risk of malnutrition in chronic liver disease $(2,3)$.

Despite the recent improvement in general nutritional status of general population, the prevalence of malnutrition in patients with chronic liver disease has not significantly decreased. Even in the era of obesity, the prevalence of malnutrition in chronic liver disease is still considerably reported with a wide variety, ranging from $10 \%$ to $100 \%$ (4). In the obesity era, a narrative review for malnutrition prevalence and method of evaluating malnutrition in patients with chronic liver disease is needed. Therefore, there is a need to verify the right value of prevalence of malnutrition.

The first issue related to malnutrition in patients with chronic liver disease is that that each study used its own diagnostic method, criteria, and cut-off value, which is the main reason for wide range of malnutrition prevalence in previous studies. Previously, each study used a wide variety of diagnostic tools and different cut off values even when using the same diagnostic tool. Various approaches used comprised of subjective global assessment (SGA), mid arm muscle circumference (MAMC), triceps skinfold thickness (TSF), and handgrip strength (HGS); however, each study used different criteria $(1,5)$. In addition, cut-off values to define malnutrition also varied to a great extent even in some cases where the same evaluation tool was used.

Second issue is that recently non-alcoholic fatty liver has become the most common cause of chronic liver disease. Therefore, it is necessary to narrative review the changes in prevalence and characteristics of malnutrition according to changes in etiology of chronic liver disease. But most of studies were performed 20 years ago and there is an unmet need in this area. Traditionally, heavy alcohol consumption and chronic viral infection were the main reasons of chronic liver diseases; however, more recently nonalcoholic fatty liver disease (NAFLD) closely associated with obesity is the leading cause of chronic liver disease (5). However, studies on malnutrition prevalence in patients with chronic liver disease in the obese era are limited. In the overnutrition era, further research is required to determine the malnutrition prevalence in patients with chronic liver diseases and its clinical characteristics. Also, majority of the studies which were conducted 10-30 years ago and had limited observations i.e., $<100$ patients (6). In this paper, most reference studies on liver disease prevalence associated with malnutrition dated prior to 2000, and studies on chronic liver disease caused by NAFLD after 2000 until recently were extremely scarce, when obesity became a big public health issue.

This study aimed to critically appraise the prevalence of malnutrition according to diagnostic tools, etiology and time in obesity era.

We present the study in accordance with the Narrative Review reporting checklist (available at http://dx.doi. org/10.21037/atm-20-4868).

\section{Methods}

\section{Database}

Literature review of PubMed and EMBASE studies published between 1980 and 2020 was conducted. Key words were malnutrition, undernutrition, cachexia, marasmus, nutrition, liver, and cirrhosis, and then the prevalence was added and re-searched within each result. Duplicated data from search resources were excluded using the study title. After checking the title and abstract, the full manuscripts were reviewed. Some data were extracted by examining the cited texts of the original article.

\section{Definition of malnutrition and nutrition assessment tool (NAT)}

To date, about 32 different definitions of malnutrition were introduced (1). In most papers, the NAT, and the nutrition screening tool (NST) have been confusingly used. NST usually uses questionnaires (malnutrition screening tool, mini-nutritional assessment, malnutrition universal screening tool, nutrition risk screening), while NAT uses MAMC, TSF, hand grip, SGA, and imaging devices (bioelectrical impedance analysis, CT, MRI). Among them SGA, anthropometric method [mid-arm muscular area (MAMA), TSF], and clinical judgement are the most frequently used as the definition of malnutrition.

\section{$S G A$}

SGA uses dietary habits of patients, weight loss in previous 
6 months, and information regarding gastrointestinal symptoms to assess malnutrition. Weight change is evaluated by proportionate and absolute loss $(\mathrm{kg})$. Evaluation criteria of malnutrition measured by weight change are as follows: "small loss" stands for weight loss of $<5 \%$; "potentially significant loss" for weight loss from $5 \%$ to $10 \%$; and "definitely significant loss" for weight loss of $>10 \%$. Simultaneously, evaluation criteria include dietary pattern changes, gastrointestinal symptoms, comorbidities, and physical examination results. Physical examination is performed to obtain information on subcutaneous fat loss (triceps, chest), muscle wasting (quadriceps and deltoids), edema, and ascites. Each part is assigned a score of normal $(0)$, mild $(+1)$, moderate $(+2)$, or severe $(+3)$ based on the degree of severity. However, since most of the evaluation items are subjective, there may be variations among observers (inter, and intra-observer variation). SGA classes are categorized into A, B, and C, where A is judged as "well nourished," B "moderately (or suspected to be) malnourished," and C "severely malnourished." SGA is advantageous because it enables doctors or trained healthcare professionals suggest clear treatment plans suitable for patients by examining them in various aspects and that it is a simple and cost effective method compared to other assessment options (7).

\section{Anthropometric tool}

MAMA, MAMC, and TSF are more widely used than other methods in anthropometric tool category (4). MAMC is performed on a patient's right arm and is measured in the middle position between acromion and olecranon (7). The equation used to define MAMA is different according to sex: $\left[(\mathrm{MAC}-\varpi \times \mathrm{TSF})^{2} / 4 \varpi\right]-10$ for men and [MAC $\left.-\varpi \times \mathrm{TSF}^{2} / 4 \varpi\right]-6.5$ for women (8). However, MAMC and MAMA are used interchangeably with no distinction in meaning in some studies (1). Low and high cut-off values for MAMA were identified as $\leq 27.3$ and $\geq 29.6 \mathrm{~cm}$, respectively, for men, and $\leq 22.3$ and $\geq 24.6 \mathrm{~cm}$, respectively, for women (9). However, different cut-off values are often used by researchers and by regions. TSF is calculated as the mean of three skin fold measurements using the Lange skinfold caliper midway between acromion and the tip of olecranon (7). Furthermore, relative cut-off values compared to healthy individuals rather than absolute values are often used in TSF method (10).

\section{Clinical judgment}

Malnutrition was defined according to the clinical judgment that often lacked presentation of definite diagnosis criteria in some studies (11).

\section{Results}

\section{Basic characteristics of 16 published studies}

The literature review was performed using a total of sixteen studies published between 1980 and 2020 regarding malnutrition in patients with chronic liver disease (Table 1) (4,7,12-25). Among the sixteen studies, three were conducted in the 1980s, six in the 1990s, five in the 2000s, and two in the 2010s. The proportion of cirrhotic patients among the sixteen studies accounted for approximately $20.2-100 \%$, and the overall malnutrition prevalence was approximately $40.7 \%$ (10-85\%). Ten studies were published from the West (Europe and North America), three from South America (Brazil), and three from Asia (India and South East Asia). Each study used various diagnostic tools to assess malnutrition. Anthropometric, clinical, and SGA methods were widely used among the diagnostic tools. Anthropometric method was highly used by eleven studies, followed by clinical judgment by seven and SGA diagnostic tool by five studies. In fifteen studies excluding a study that used medical records, the overall malnutrition prevalence ranged from $10 \%$ to $80.3 \%$, with an average of $36.4 \%$. Malnutrition prevalence according to the diagnostic tool was approximately $28-85 \%$ for SGA, $30.8-78.5 \%$ for the anthropometric approach, and $21-80.3 \%$ for the clinical judgment.

\section{Malnutrition prevalence according to diagnostic methods}

Various diagnostic tools were used to assess malnutrition in each study. Most of the studies used two or more diagnostic tools. Four studies used three or more tools, whereas seven studies used two diagnostic tools at the same time. In five studies, only one diagnostic tool (clinical judgment) was used (Table 2). Only eleven studies used one or more objective assessment tools to diagnose malnutrition. Among the sixteen studies, eleven showed malnutrition prevalence using anthropometric methods, seven used clinical judgment, and five used SGA method. Malnutrition prevalence assessed by SGA ranged from 


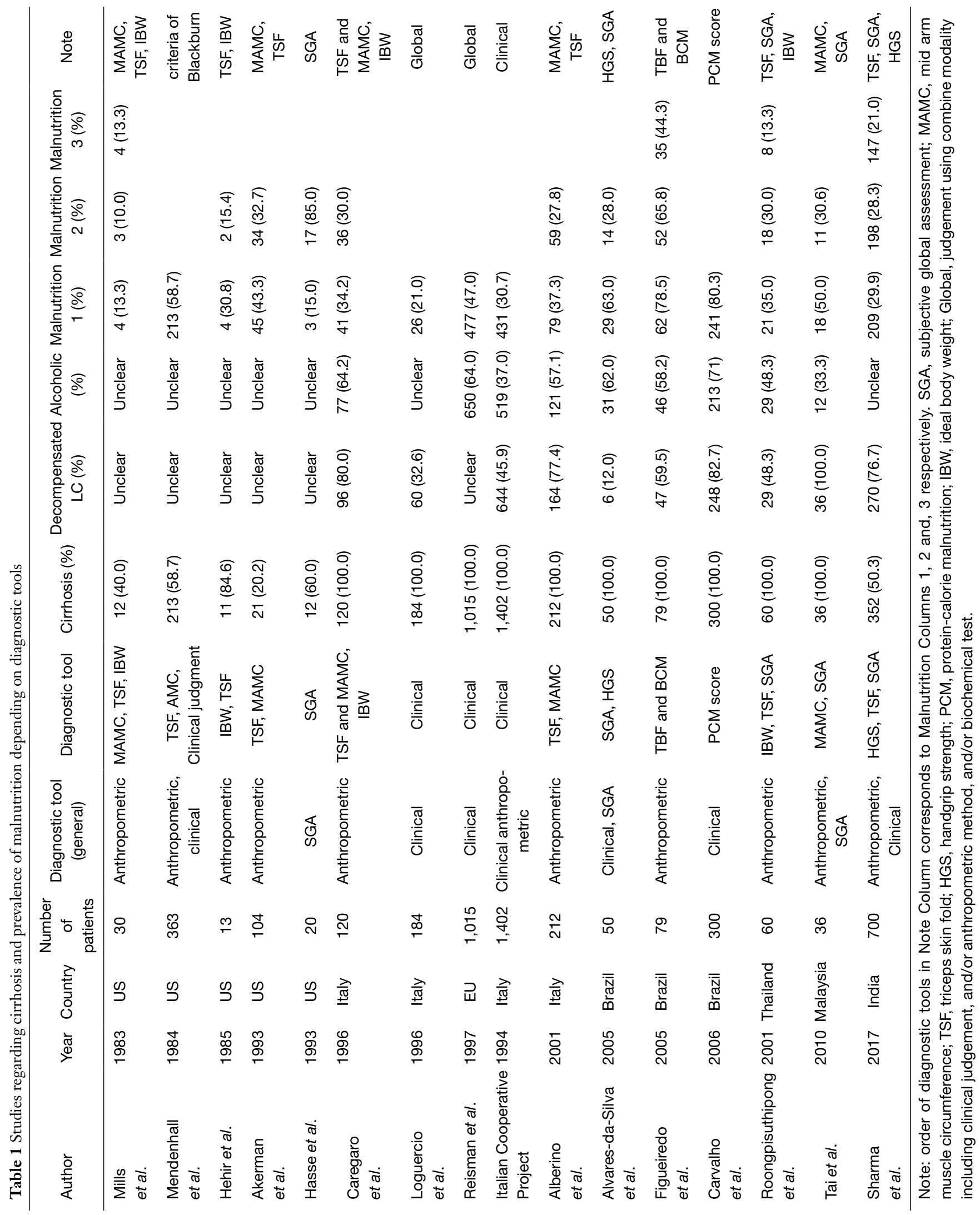


Table 2 Prevalence of malnutrition classified by diagnostic tools

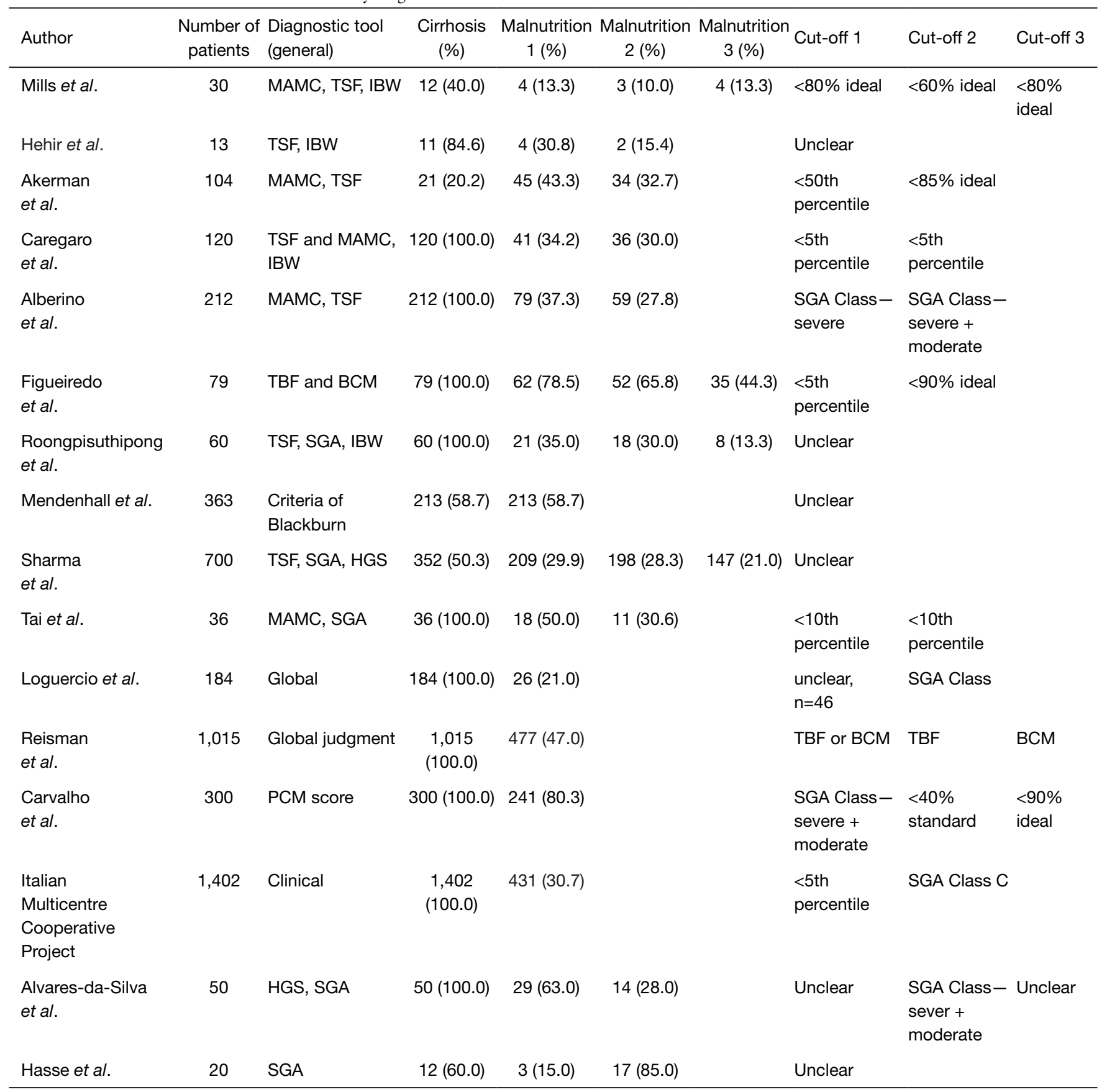

SGA, subjective global assessment; MAMC, mid arm muscle circumference; TSF, triceps skin fold; HGS, handgrip strength; PCM, protein-calorie malnutrition; IBW, ideal body weight; TBF, total body fat; BCM, body cell mass; Global, judgement using combine modality including clinical judgement, and/or anthropometric method, and/or biochemical test.

$28 \%$ to $85 \%$ (mean, $29.8 \%$ ). Several anthropometric methods were available to assess malnutrition. Malnutrition prevalence was $13.3-50 \%$ (mean, $36.3 \%$ ) for MAMC, $10-35 \%$ (mean, $29.9 \%$ ) for TSF, and $21-63 \%$ (mean,
$23.5 \%)$ for handgrip. Malnutrition prevalence resulting from clinical judgment was $21-80.3 \%$ (mean, $40.5 \%$ ) (7,12-15,17,23-25). Handgrip showed the lowest malnutrition prevalence rate as opposed to non-SGA 
Table 3 Prevalence of malnutrition in researches on cirrhosis patients only

\begin{tabular}{|c|c|c|c|c|c|c|}
\hline Author & $\begin{array}{l}\text { Number of } \\
\text { patients }\end{array}$ & $\begin{array}{l}\text { Cirrhosis } \\
(\%)\end{array}$ & $\begin{array}{c}\text { Malnutrition } \\
1(\%)\end{array}$ & $\begin{array}{c}\text { Malnutrition } \\
2(\%)\end{array}$ & $\begin{array}{c}\text { Malnutrition } \\
3(\%)\end{array}$ & Note \\
\hline Caregaro et al. & 120 & $120(100.0)$ & $41(34.2)$ & $36(30.0)$ & & Anthropometric (TSF and MAMC), IBW \\
\hline Reisman et al. & 1,015 & $1,015(100.0)$ & $477(47.0)$ & & & Global judgment (clinical, and anthropometric) \\
\hline Alberino et al. & 212 & $212(100.0)$ & $79(37.3)$ & $59(27.8)$ & & MAMC, TSF \\
\hline $\begin{array}{l}\text { Alvares-da-Silva } \\
\text { et al. }\end{array}$ & 50 & $50(100.0)$ & $29(63.0)$ & $14(28.0)$ & & HGS, SGA \\
\hline Figueiredo et al. & 79 & $79(100.0)$ & $62(78.5)$ & $52(65.8)$ & $35(44.3)$ & SGA, TBF and BCM \\
\hline Carvalho et al. & 300 & $300(100.0)$ & $241(80.3)$ & & & PCM score \\
\hline
\end{tabular}

SGA, subjective global assessment; MAMC, mid arm muscle circumference; TSF, triceps skin fold; HGS, handgrip strength; PCM, protein-calorie malnutrition; IBW, ideal body weight; Global, judgement using combine modality including clinical judgement, and/or anthropometric method, and/or biochemical test.

Table 4 Prevalence of malnutrition in patients with decompensated liver cirrhosis

\begin{tabular}{|c|c|c|c|c|c|}
\hline Author & Number of patients & Cirrhosis (\%) & Decompensated LC (\%) & Malnutrition (\%) & Diagnostic tool \\
\hline Alberino et al. & 212 & $212(100.0)$ & $164(77.4)$ & $66(40.2)$ & MAMC, TSF \\
\hline Figueiredo et al. & 79 & $79(100.0)$ & $47(59.5)$ & $44(93.6)$ & TBF and BCM \\
\hline Carvalho et al. & 300 & $300(100.0)$ & $248(82.7)$ & $216(87.1)$ & PCM score \\
\hline
\end{tabular}

MAMC, mid arm muscle circumference; TSF, triceps skin fold; TBF, body fat mass; BCM, body cell mass; PCM, protein-calorie malnutrition; Global, judgement using combine modality including clinical judgement, and/or anthropometric method, and/or biochemical test.

clinical judgment with the highest prevalence (7). When we looked at the malnutrition prevalence according to various diagnostic tools, clinical judgement method showed higher malnutrition prevalence than handgrip method.

\section{Malnutrition prevalence in compensated and decompensated cirrbosis}

Ten out of sixteen studies exclusively included cirrhotic patients (Table 3). Malnutrition prevalence in cirrhosis in these ten studies was $13.3-80.3 \%$ (mean, 39.9\%) (4,17-25), and the number of patients varied from as little as
36 patients up to a maximum of 1,402 patients $(4,17-25)$. In four studies, malnutrition prevalence was presented for decompensated liver cirrhosis (LC) (Child B + Child C) patients (Table 4) $(4,18,21,23)$. Malnutrition prevalence among the decompensated LC patients in these 4 studies ranged from $26.7 \%$ to $93.6 \%$ (mean, $44.1 \%$ ).

\section{Malnutrition prevalence in patients with alcohol-related cirrbosis}

All sixteen studies included patients with various causes of liver disease. Most of those did not suggest malnutrition prevalence according to liver disease etiology, and only 
Table 5 Prevalence of malnutrition according to etiology (alcoholic vs. HCV)

\begin{tabular}{|c|c|c|c|c|c|c|c|c|}
\hline Author & $\begin{array}{l}\text { Number of } \\
\text { patients }\end{array}$ & $\begin{array}{l}\text { Alcoholic } \\
(\%)\end{array}$ & HCV (\%) & $\begin{array}{c}\text { Malnutrition } \\
1(\%)\end{array}$ & $\begin{array}{c}\text { Malnutrition } \\
2(\%)\end{array}$ & Diagnostic tool & Cut-off 1 & Cut-off 2 \\
\hline Reisman et al. & 1,015 & $650(64.0)$ & & $306(47.0)$ & & Global judgment & Unclear & \\
\hline $\begin{array}{l}\text { Roongpisuthipong } \\
\text { et al. }\end{array}$ & 60 & $29(48.3)$ & & $3(10.3)$ & & SGA & Child C & \\
\hline Carvalho et al. & 300 & $213(71.0)$ & & $158(74.2)$ & $106(49.8)$ & PCM score, TSF & Unclear & $50 \%$ loss of fat \\
\hline $\begin{array}{l}\text { Italian Cooperative } \\
\text { Project }\end{array}$ & 1,402 & & 369 (26.3) & $84(22.9)$ & $91(24.6)$ & MAMA, MAFA & Unclear & Unclear \\
\hline Tai et al. & 36 & & $15(41.7)$ & $4(26.7)$ & & SGA & SGA Class C & \\
\hline
\end{tabular}

SGA, subjective global assessment; MAMA, mid arm muscle area; MAMC, mid arm muscle circumference; TSF, triceps skin fold; PCM, protein-calorie malnutrition; Global, judgement using combine modality including clinical judgement, and/or anthropometric method, and/or biochemical test.

five studies presented malnutrition prevalence according to hepatic disease etiology $(4,19,20,24,25)$. In these five studies, liver disease was divided into alcoholic and non-alcoholic, and two studies were identified to have exclusively targeted HCV-infected patients $(20,25)$ (Table 5). Malnutrition prevalence was 38.2\% (10.3-75\%) in alcoholic liver disease and $23.7 \%$ (22.9-26.7\%) in HCV-related liver disease. Malnutrition prevalence was $14.5 \%$ higher in patients with alcohol-related liver disease than that in patients with HCV-related liver disease. However, no study has investigated the malnutrition prevalence in nonalcoholic fatty liver disease.

\section{Malnutrition prevalence over time}

Nine of sixteen studies were published before 2000 (12-15,17-20), and the other seven studies were published after 2000 (4,7,21-25). Malnutrition prevalence in studies published prior to 2000 ranged between $13.3 \%$ and $85 \%$ (mean, 37.6\%), whereas studies published after 2000 had a malnutrition prevalence of $13.3-78.5 \%$ (mean, $35.2 \%$ ).

\section{Discussion}

In chronic liver diseases, the average malnutrition prevalence measured using the diagnostic tools was $36.4 \%$ $(10-80.3 \%)$ in patients with all types of liver diseases. Malnutrition prevalence in patients with compensated and decompensated cirrhosis was $39.9 \%$ and $44.1 \%$, respectively. Malnutrition prevalence greatly varied depending on the diagnostic tool where non-standardized clinical judgment showed a tendency to report high malnutrition prevalence. As for the anthropometric method, malnutrition prevalence reported from MAMC was higher than TSF and handgrip, respectively.

The main reasons for such a big difference of malnutrition prevalence in patients with chronic liver disease seem to be explained by various diagnostic tools and cut-off values used. In particular, in eight studies malnutrition judgment was either ambiguous or no judgement at all. Such non-standardized clinical judgment is problematic because it uses an excessively wide definition of malnutrition resulting in a high prevalence rate.

In studies on malnutrition prevalence in patients with chronic liver disease, the anthropometric method was the most frequently used diagnostic approach, with TSF and MAMC methods being the most favored ones among them. In the event where the MAMC diagnostic tool was used, malnutrition prevalence was slightly higher than those when the TSF method was used (36.3\% vs. 29.9\%) (21). This implies the possibility that malnutrition prevalence identified by MAMC may be overestimated. In addition, another study on patients with cirrhosis demonstrated that malnutrition prevalence from MAMC method was higher than that of TSF method $(21,22)$. The reason why MAMC's malnutrition evaluation method seemed to be subjective is 
the big deviation according to the measuring person and non-uniform cut-off value.

Conversely, the actual prevalence rates for patients assessed using the SGA method range from $20 \%$ to $30 \%$ and maintained at the similar level. SGA assessment is widely used, and one of its advantages is the many validations performed for other diseases. SGA also showed good agreement with TSF (24). However, performing SGA requires more time than the anthropometric tool, and there is a risk that mild malnutrition may be ignored. For these reasons, malnutrition prevalence measured with SGA is at least 5\% lower compared to other assessment methods (non-standardized clinical judgment: mean, $40.5 \%$; MAMC: $36.3 \%$; and SGA: $29.8 \%$ ). SGA is nonetheless advantageous because it can be used together with other assessment methods, and its low variance is another strong point compared to other methods. Therefore, combined use of TSF and SGA is expected lead to a malnutrition assessment tool that can demonstrate a higher level of accuracy.

The second issue of malnutrition assessment was the different cut-off values of diagnostic tools in each study. Among these studies which used the same TSF diagnostic tool, Mills et al. used $<60 \%$ of the ideal value as cut off value for malnutrition; however, Akerman et al. used $85 \%$ as cut-off value of malnutrition $(12,15)$. Thus, the cut-off values used to determine malnutrition prevalence were different depending on researchers.

Some studies have reported that malnutrition prevalence varies according to liver disease etiology (26). Alcoholic liver disease is generally associated with high malnutrition prevalence (26). Tai et al. (25) suggested that malnutrition prevalence in patients with alcoholic liver disease is $25 \%$ higher than other liver diseases. However, Reisman et al. (19) reported that malnutrition prevalence was not different according to etiology, and if any, is negligible. Our review showed that the prevalence of malnutrition in alcoholic liver disease was $14.5 \%$ higher than that of $\mathrm{HCV}$ related liver disease (alcoholic: $38.26 \%$ vs. HCV 23.77\%) $(4,19,20,24,25)$.

Despite the overall improvement of nutritional status since 2000, malnutrition incidence reported in the literature did not decrease to a great extent. The trend observed for the period between the 1980s and 2000s, in particular, did not decrease (before 2000: 37.56\% vs. 2000 and onward: $35.19 \%)$. Therefore, malnutrition is no longer referred as a simple nutritional deficiency; rather, it requires further research on pathogenesis related to disorders created in the process of hepatic metabolism. Since 2010, more attention has been given to sarcopenia, due to reduced muscles, than has been given to adipopenia, due to energy intake deficiency, which was traditionally regarded important. For these reasons, more studies tend to evaluate sarcopenia by measuring the muscle mass rather than using traditional malnutrition assessment tools, such as SGA and TSF. Although sarcopenia is an important element in malnutrition, whether it shall be deemed as malnutrition or not remains controversial. In addition, some malnutrition findings between the East and West showed similar characteristics $(24,25)$; however, other studies on populations in the rest of the West or South America suggested different findings, which calls for the need for further research.

In conclusion, malnutrition prevalence was still considerable even in the obesity era. However, most of these studies were conducted before 2010 with a limited number of studies after 2010. It is thought that a deeper consideration is required for malnutrition prevalence and its clinical characteristics in patients with chronic liver disease in the obesity era.

\section{Acknowledgments}

Funding: This study was supported by National Research Foundation of Korea (NRF-2017R1D1A3B04033457). The funding source had no role in the study design and implementation; data collection, analysis, and interpretation; or preparation, review, or approval of the manuscript.

\section{Footnote}

Reporting Checklist: The authors have completed the Narrative Review reporting checklist. Available at http:// dx.doi.org/10.21037/atm-20-4868

Conflicts of Interest: All authors have completed the ICMJE uniform disclosure form (available at http://dx.doi. org/10.21037/atm-20-4868). The authors have no conflicts of interest to declare.

Ethical Statement: The authors are accountable for all aspects of the work in ensuring that questions related to the accuracy or integrity of any part of the work are appropriately investigated and resolved.

Open Access Statement: This is an Open Access article distributed in accordance with the Creative Commons Attribution-NonCommercial-NoDerivs 4.0 International 
License (CC BY-NC-ND 4.0), which permits the noncommercial replication and distribution of the article with the strict proviso that no changes or edits are made and the original work is properly cited (including links to both the formal publication through the relevant DOI and the license). See: https://creativecommons.org/licenses/by-nc-nd/4.0/.

\section{References}

1. Ney M, Li S, Vandermeer B, et al. Systematic review with meta-analysis: Nutritional screening and assessment tools in cirrhosis. Liver Int 2020;40:664-73.

2. Cheung K, Lee SS, Raman M. Prevalence and mechanisms of malnutrition in patients with advanced liver disease, and nutrition management strategies. Clin Gastroenterol Hepatol 2012;10:117-25.

3. Juakiem W, Torres DM, Harrison SA. Nutrition in cirrhosis and chronic liver disease. Clin Liver Dis 2014;18:179-90.

4. Carvalho L, Parise ER. Evaluation of nutritional status of nonhospitalized patients with liver cirrhosis. Arq Gastroenterol 2006;43:269-74.

5. European Association for the Study of the Liver; European Association for the Study of the Liver. EASL Clinical Practice Guidelines on nutrition in chronic liver disease. J Hepatol 2019;70:172-93.

6. Plauth M, Bernal W, Dasarathy S, et al. ESPEN guideline on clinical nutrition in liver disease. Clin Nutr 2019;38:485-521.

7. Sharma P, Rauf A, Matin A, et al. Handgrip Strength as an Important Bed Side Tool to Assess Malnutrition in Patient with Liver Disease. J Clin Exp Hepatol 2017;7:16-22.

8. Heymsfield SB, McManus C, Smith J, et al. Anthropometric measurement of muscle mass: revised equations for calculating bone-free arm muscle area. Am J Clin Nutr 1982;36:680-90.

9. Wu LW, Lin YY, Kao TW, et al. Mid-arm muscle circumference as a significant predictor of allcause mortality in male individuals. PLoS One 2017;12:e0171707.

10. Rodrigues J, Santin F, Brito F, et al. Nutritional status of older patients on hemodialysis: Which nutritional markers can best predict clinical outcomes? Nutrition 2019;65:113-9.

11. Sam J, Nguyen GC. Protein-calorie malnutrition as a prognostic indicator of mortality among patients hospitalized with cirrhosis and portal hypertension. Liver
Int 2009;29:1396-402.

12. Mills PR, Shenkin A, Anthony RS, et al. Assessment of nutritional status and in vivo immune responses in alcoholic liver disease. Am J Clin Nutr 1983;38:849-59.

13. Mendenhall CL, Anderson S, Weesner RE, et al. Proteincalorie malnutrition associated with alcoholic hepatitis. Veterans Administration Cooperative Study Group on Alcoholic Hepatitis. Am J Med 1984;76:211-22.

14. Hehir DJ, Jenkins RL, Bistrian BR, et al. Nutrition in patients undergoing orthotopic liver transplant. JPEN J Parenter Enteral Nutr 1985;9:695-700.

15. Akerman PA, Jenkins RL, Bistrian BR. Preoperative nutrition assessment in liver transplantation. Nutrition 1993;9:350-6.

16. Hasse J, Strong S, Gorman MA, et al. Subjective global assessment: alternative nutrition-assessment technique for liver-transplant candidates. Nutrition 1993;9:339-43.

17. Caregaro L, Alberino F, Amodio P, et al. Malnutrition in alcoholic and virus-related cirrhosis. Am J Clin Nutr 1996;63:602-9.

18. Loguercio C, Sava E, Sicolo P, et al. Nutritional status and survival of patients with liver cirrhosis: anthropometric evaluation. Minerva Gastroenterol Dietol 1996;42:57-60.

19. Reisman Y, Gips CH, Lavelle SM. Assessment of liver cirrhosis severity in 1015 patients of the Euricterus database with Campbell-Child, Pugh-Child and with ascites and ascites-nutritional state (ANS) related classifications. Euricterus Project Management Group. Hepatogastroenterology 1997;44:1376-84.

20. Nutritional status in cirrhosis. Italian Multicentre Cooperative Project on Nutrition in Liver Cirrhosis. J Hepatol 1994;21:317-25.

21. Alberino F, Gatta A, Amodio P, et al. Nutrition and survival in patients with liver cirrhosis. Nutrition 2001;17:445-50.

22. Alvares-da-Silva MR, Reverbel da Silveira T. Comparison between handgrip strength, subjective global assessment, and prognostic nutritional index in assessing malnutrition and predicting clinical outcome in cirrhotic outpatients. Nutrition 2005;21:113-7.

23. Figueiredo FA, De Mello Perez R, Kondo M. Effect of liver cirrhosis on body composition: evidence of significant depletion even in mild disease. J Gastroenterol Hepatol 2005;20:209-16.

24. Roongpisuthipong C, Sobhonslidsuk A, Nantiruj K, et al. Nutritional assessment in various stages of liver cirrhosis. Nutrition 2001;17:761-5.

25. Tai ML, Goh KL, Mohd-Taib SH, et al. Anthropometric, 
Page 10 of 10

biochemical and clinical assessment of malnutrition in Malaysian patients with advanced cirrhosis. Nutr J 2010;9:27.

26. Caly WR, Strauss E, Carrilho FJ, et al. Different degrees

Cite this article as: Shin S, Jun DW, Saeed WK, Koh DH. A narrative review of malnutrition in chronic liver disease. Ann Transl Med 2021;9(2):172. doi: 10.21037/atm-20-4868
Shin et al. Prevalence of malnutrition in liver disease

of malnutrition and immunological alterations according to the aetiology of cirrhosis: a prospective and sequential study. Nutr J 2003;2:10. 